\title{
Facteurs Associés à la Réticence de la Vaccination Contre la Poliomyélite chez les Mères dans la Commune d'Abobo, Abidjan: Cas du Quartier Samaké
}

\author{
Yéboua Yao David, \\ Tia Félicien Yomi, \\ Koffi Olivier Yao,
}

Doctorant en Anthropobiologie Institut des Sciences Anthropologiques de Développement (ISAD) UFR SHS, Université Félix Houphouët-Boigny Abidjan

Kouamé Atta,

Maître de Conférences en Anthropobiologie, Institut des Sciences

Anthropologiques de Développement (ISAD), Université Félix HouphouëtBoigny-Abidjan

\section{Yoro Marcel Blé,}

Professeur Titulaire en Socio-anthropologie de la Santé, Institut des Sciences Anthropologiques de Développement (ISAD), Université Félix HouphouëtBoigny-Abidjan

Kone Drissa,

Professeur Titulaire de Psychiatrie, UFR des Sciences Médicales d'Abidjan, Université Félix Houphouët-Boigny-Abidjan

\section{Résumé}

Cette étude porte sur les facteurs associés à la réticence de la vaccination contre la poliomyélite chez les mères dans la commune d'Abobo à Abidjan. Elle a été réalisée à partir d'une approche qualitative basée sur des entretiens semi-directifs auprès de trente (30) mères ayant en charge un enfant de 0 à 5 ans au quartier Samaké de ladite commune. Il ressort de l'analyse des résultats, l'association de plusieurs facteurs dans la réticence à la vaccination contre poliomyélite. Les facteurs incriminés sont la mauvaise compréhension et connaissance de la maladie et de la vaccination, l'ignorance, l'inquiétude concernant la sécurité du vaccin, le manque d'information et les sources d'influence, notamment celle des proches, ou des amies. Des stratégies axées sur une sensibilisation accrue de la population de cette maladie sont autant de 
facteurs qui contribueront à l'acceptation de la vaccination et l'éradication de la poliomyélite en Côte d'Ivoire.

Mots-clés: Réticence, Poliomyélite, Milieu urbain, Abidjan

\title{
Factors Associated with the Reaction of Poliomyelitis Vaccination Among Mothers in the Abobo Community, Abidjan: Case of the Samaké District
}

\author{
Yéboua Yao David, \\ Tia Félicien Yomi, \\ Koffi Olivier Yao,
}

Doctorant en Anthropobiologie Institut des Sciences Anthropologiques de

Développement (ISAD) UFR SHS, Université Félix Houphouët-Boigny

Abidjan

Kouamé Atta,

Maître de Conférences en Anthropobiologie, Institut des Sciences

Anthropologiques de Développement (ISAD), Université Félix Houphouët-

Boigny-Abidjan

Yoro Marcel Blé,

Professeur Titulaire en Socio-anthropologie de la Santé, Institut des Sciences Anthropologiques de Développement (ISAD), Université Félix Houphouët-

Boigny-Abidjan

Kone Drissa,

Professeur Titulaire de Psychiatrie, UFR des Sciences Médicales d'Abidjan, Université Félix Houphouët-Boigny-Abidjan

\section{Abstract}

This paper focuses on examining the factors associated with the reluctance to vaccination against poliomyelitis in mothers living in the city of Abobo in Abidjan. It was carried out on the basis of a qualitative approach based on semi-structured interviews with thirty (30) mothers in care of one child aged of 0 to 5 in Samaké district of the same city. The analysis of the results showed the association of several factors in the reluctance to vaccination against polio. The incriminated factors include poor understanding and knowledge of the disease and vaccination, ignorance, concern about vaccine safety, lack of information, and sources of influence 
from relatives or friends. Strategies for increased awareness of the population concerning this disease refer to all the factors that contribute to the acceptance of vaccination and the eradication of polio in Côte d'Ivoire.

Keywords: Reluctance, Poliomyelitis, Urban environment, Abidjan

\section{Introduction}

La poliomyélite est une maladie infectieuse et invalidante dont l'origine remonte depuis l'antiquité. Elle est causée par un virus appelé «poliovirus », appartenant au genre Entérovirus et issu de la famille des Picornaviridae (Blondel et al., 2000). Les entérovirus forment en effet, un groupe hétérogène avec des virus provoquant des pathologies totalement bénignes, responsables d'infections rhino-pharyngées (par exemple les rhinovirus), mais aussi des infections plus graves, telles que des péricardites dues à des coxsackievirus.

Plus d'un siècle après sa découverte en 1855, le virus de la poliomyélite continue de sévir dans le monde. Selon les données épidémiologiques, au moment de la mise en place du projet d'éradication de la maladie en 1988, l'Organisation Mondiale de la Santé (OMS) estimait qu'il y avait plus de 350000 nouveaux cas de poliomyélite paralytique chaque année et dénombrait plus de 125 pays endémiques (Antona \& Guerin, 2005).

Actuellement, le Pakistan et l'Afghanistan restent deux pays dans lesquels les poliovirus sauvages sont très actifs. Des cas isolés de PVDV (Poliovirus Dérivé du Vaccin) sont également signalés dans certains pays. Par exemple, au Cameroun et en Guinée Équatoriale, des cas de PVDV de type 2 ont également été confirmés. Au Nigéria, l'incidence de PVDV de type 2 est passée de 4 à 30 nouveaux cas de 2013 à 2014. D'autres cas ont aussi été signalés sur l'île de Madagascar, en Ukraine et en Asie du Sud-Est (Tan Sien, 2016).

La poliomyélite fait partir des fléaux de l'humanité dont il n'existe, à ce jour, aucun traitement antiviral curatif. Les mesures de prévention (le respect strict des règles hygiéno-diététiques, la soumission à certaines actions de dépistage et la vaccination) restent les seules méthodes efficaces pour lutter contre cette maladie (Haut Conseil de la Santé Publique, 2014).

Après l'expansion mondiale de la vaccination anti poliomyélite, le nombre de pays atteints par cette maladie a considérablement diminué. L'incidence de la maladie a chuté de plus de $99 \%$, avec seulement 359 cas notifiés pour l'année 2014 (OMS, 2014). Cependant, elle persistait dans trois pays endémiques tels que: le Pakistan, l'Afghanistan et le Nigeria (OMS, 2015). La couverture mondiale reflète la proportion d'enfants qui ont reçu trois doses de vaccin anti-poliomyélite. Elle est globalement considérée comme stable, mais inégale selon les régions. En 2014, 86\% des nourrissons ont reçu 
trois doses de vaccin antipoliomyélitique (OMS, 2014), alors qu'en 2011, 22,4 millions d'enfants dans le monde n'avaient pas du tout, ou que partiellement, accès à des vaccins. À ce jour, l'OMS estime qu'environ 19,5 millions de nourrissons ne bénéficient pas de la vaccination dans le monde, ce qui montre une nette amélioration de la couverture vaccinale en quelques années. Or, des efforts restent à fournir dans de nombreux pays, notamment en Afrique et en Asie du sud-est (OMS, 2017).

La Côte d'Ivoire à l'instar de tous les pays a mis en place un programme de surveillance épidémiologique qui organise des campagnes de vaccination contre la poliomyélite pour éradiquer cette maladie. Les efforts consentis par l'État ivoirien ont permis d'accomplir des progrès notables dans l'éradication de cette maladie. Ces progrès sont dus à la couverture vaccinale et le système de surveillance épidémiologique. Cependant, force est de constater qu'il y a une persistance des cas de paralysie dus au poliovirus sauvage et des poches de résistances, provoquant ainsi des incapacités physiques (DCPEV, 2017) avec une prévalence nationale de couverture de 95\% et 13 cas de PFA (Paralysie Flasques Aigue) détectés.

Cette situation pourrait être affiliée à un état de réticence qui s'est emparé d'une partie de la population. On assiste dès lors, à un nombre d'enfants qui n'ont pas reçu le vaccin au cours des programmes de vaccinations. Selon les données de la Direction de Coordination du Programme Élargi de Vaccination (DCPEV), concernant la Journée Nationale de Vaccination contre la Poliomyélite du 24 au 27 mars 2017, dans la région sanitaire d'Abidjan, la proportion d'enfants de 0 à 5 ans qui ont reçu le vaccin était estimée à un million trois cent quatorze milles cent un (1314101) sur un total d' un million quatre cent vingt-cinq cinq cent soixante-douze (1425572) enfants. Il s'agit ici de cent onze mille quatre cent soixante-onze (111471) enfants qui n'ont pas reçu le vaccin contre la poliomyélite (DCPEV, 2017).

Ce résultat semble montrer l'existence d'un goulot d'étranglement qui empêcherait la continuité dans l'utilisation des services de vaccination. La commune d'Abobo s'illustre négativement dans cette situation avec un taux de couverture vaccinale de $80 \%$ et un nombre de 79202 enfants qui n'ont pas reçu le vaccin contre la poliomyélite et $55 \%$ dans le quartier Samaké (DCPEV, 2017).

Dès lors nous nous demandons : pourquoi malgré tous les efforts consentis par la Côte d'Ivoire et ses partenaires internationaux dans l'éradication de la poliomyélite, plusieurs enfants âgés de 0 à 5 ans ne sont pas vaccinés dans le quartier Samaké de la commune d'Abobo ? Autrement dit, quels sont les facteurs associés à la réticence de la vaccination contre la poliomyélite chez les mères d'enfants âgés de 0 à 5 ans dans le quartier Samaké de la commune d'Abobo ? 


\section{Dispositif Méthodologique}

L'étude s'est déroulée du 20 juillet au 19 août 2017 au quartier Samaké dans la commune d'Abobo à Abidjan (Côte d'Ivoire). Ce quartier est l'un des aires sanitaires que constitue le district sanitaire d'Abobo Ouest. Dans cette étude, nous avons mobilisé la méthode qualitative de collecte et d'analyse des données. Ce choix méthodologique est motivé par notre conviction que les facteurs associés à la réticence de la vaccination contre la poliomyélite chez les mères dans la commune d'Abobo à Abidjan ne sont pas plus d'ordre numérique mais plutôt relatifs à des aspects sociétaux et culturels dont l'appréhension nécessite une approche de type qualitatif (Tia et al., 2017).

Cette méthode a permis d'identifier et de comprendre ce qui influence les comportements des mères vis-à-vis de la vaccination contre la poliomyélite. Ce travail est donc le fruit d'entretiens semi-structurés réalisés avec30 ménages ayant en charge au moins un enfant de 0 à 5 ans. La stratégie de recrutement des mères était basée sur la volonté et la capacité de celles-ci à réaliser des entretiens. La technique d'échantillon de volontaires a donc été retenue dans la mesure où dans cet échantillon, « les sujets sont sélectionnés en fonction de quotas selon leur disponibilité et de leur volonté à participer à l'étude » (Lefrançois, 1991). Une correction est apportée à l'échantillon, après coup, pour ajustement (N'da, 2015).

L'entretien semi-directif choisi pour collecter dans le cadre de cette étude est un outil à la frontière de la santé et de l'anthropologie (Imbert, 2010). Avec l'appui d'une autorisation administrative du Ministère de la Santé et de l'Hygiène Publique (MSHP) et de la Direction Générale de la Santé et de l'Hygiène Publique, les entretiens ont été réalisés sur la base du consentement éclairé des mères. Ceux-ci ont été enregistrés et restent anonymes.

Les données recueillies ont été ensuite transcrites manuellement dans leur intégralité pour constituer un corpus. Chaque transcription a été soumise à l'analyse de contenu thématique dans le sens définit par Saraiva, (2014) et Yoro (2012). Cette analyse a consisté d'abord en une lecture flottante de la totalité du corpus obtenu pour saisir les messages apparents. Ensuite, l'appropriation du contenu a conduit à dégager les unités de sens. Cette phase a permis de faire un codage des entretiens ; c'est-à-dire, nous avons lié aux mots ou expressions les plus apparents des unités de sens. Enfin, tout verbatim ayant des caractères communs a été regroupé sous un titre générique conformément aux grandes thématiques retenues dans les guides d'entrevue.

L'interprétation des données a consisté d'une part, à organiser les résultats obtenus et à effectuer les inférences en vue d'évaluer le degré de validité générale de notre postulat et d'autre part, à comparer ces résultats à ceux d'autres recherches réalisées sur les facteurs de réticence à la vaccination d'autres maladies. 


\section{Résultats}

À l'analyse des données collectées, il ressort que plusieurs facteurs interagissent dans la réticence à la vaccination contre la poliomyélite chez les mères. On note entre autres, la méconnaissance de la maladie et de la vaccination, les sources d'informations et d'influence.

\section{La méconnaissance de la maladie et de la vaccination}

Plusieurs définitions émanent du discours de nos enquêtées à propos de cette maladie et de la vaccination. Certaines faisaient une description symptomatique, tandis que d'autres ignoraient ou n'avaient aucune connaissance de la poliomyélite. Elles étaient incapables de donner une définition claire de la maladie. On peut constater la façon de définir la poliomyélite dans les propos de nos enquêtées :

"Pour moi, la poliomyélite c'est lorsque les pieds des enfants sont gâtés, ils n'arrivent pas à bien marcher ou ils marchent avec des béquilles ou bien on le met dans chaise roulante, mais ce qui n'est sûr ce n'est pas une bonne maladie».

"La poliomyélite est une maladie dangereuse qui paralyse l'enfant. Elle commence par le paludisme et par la suite paralyse l'enfant. »

Une autre mère a ajouté :

"Franchement, j'entends parler de ça, mais je ne connais pas cette maladie. Hum je sais que c'est une maladie, mais je ne peux pas te dire comment on l'attrape. »

Parler de la maladie, c'est interroger son sens et sa provenance. Ainsi, la piqûre et le caractère inné de la maladie ont été les éléments majeurs qui émergeaient des propos de la plupart des enquêtées, comme le mode de transmission de la maladie. Les transcriptionsci-dessous illustrent bien notre argumentation :

"On peut contracter la maladie de plusieurs façons même chez un individu. Pour moi c'est à travers la piqûre des insectes et aussi par tous les moyens qu'on peut attraper la maladie».

Une autre interviewée a renchéri avec ses propos :

"Selon moi, j'ai entendu qu'il y a des enfants qui naissent avec la maladie, depuis qu'il est dans le ventre de sa maman, il a déjà ça».

La vaccination peut être abordée sous plusieurs angles tels que l'utilité, l'efficacité et la sécurité. Ces concepts ont servi aussi pour identifier les connaissances et les croyances des mères en lien avec la vaccination.

Toutefois, bien qu'elles reconnaissent une certaine utilité du vaccin, ces mères mentionnaient également que la vaccination était inutile, en précisant que le corps était capable de combattre les maladies grâce à la 
volonté de l'être divin «Dieu», que leurs enfants ne risquaient pas de contracter cette infection, ou encore que l'immunité naturelle est meilleure que l'immunité acquise, et que le corps est capable de lutter contre les maladies. Ces témoignages ci -après illustrent cette assertion :

"C'est vrai que c'est une bonne chose de vacciner les enfants. Avant là, nos parents ne faisaient pas vacciner les enfants. Il n'y avait pas tout ça là, mais les enfants étaient en bonne santé, parce que tout se faisait naturellement. C'est Dieu qui nous a créé, donc il a mis des choses dans notre corps pour nous protéger, c'est mieux que vaccins des blancs là !!! »

Concernant l'efficacité de la vaccination, cette dernière était remise en cause par le fait qu'il était possible de contracter la maladie même étant vacciné, ou le fait que tous les vaccins ne protègent pas contre toutes les souches du virus.

"Vraiment, on dit merci à Dieu, la vaccination a fait qu'il y a des maladies qu'on n'entend plus parler de ça, même la poliomyélite aussi, on ne parle pas trop de ça. Mais souvent, on voit des enfants qu'on vaccine et après ces mêmes enfants tombent malades et c'est la maladie contre laquelle on les avait vacciné qui est détectée. En tout cas, concernant polio là, je sais que ça existe, j'entendu parler de ça, mais pour moi c'est du bla-bla quoi !!!»

La durée de l'efficacité est également remise en cause. Une mère évoque cela en parlant du vaccin contre la poliomyélite.

«Je ne pense pas que le vaccin soit efficace. Sinon, pourquoi il allait faire ça à chaque moment? Même vaccination là, on peut faire ça deux ou trois fois dans l'année, c'est que ce n'est pas efficace. Si c'était le cas, une fois qu'on vaccine l'enfant, on ne doit plus reprendre encore. "

Lorsqu'on parle de vaccination, l'aspect de la sécurité est très important à évoquer. Par contre, certaines mères ont souligné leurs inquiétudes concernant la sécurité des vaccins par les agents vaccinateurs.

"Je n'accepte pas que les agents vaccinateurs vaccinent mon enfant, je pense que ces agents ne sont même pas qualifiés et ils ne conservent pas bien et ne prennent pas soin du matériel de vaccination. Ce sont des jeunes qu'on ramasse dans les quartiers et qui ne maîtrisent rien du tout. Vraiment ça fait que je ne suis pas à l'aise, je préfère dans ce cas me rendre à l'hôpital.»

Ces informations montrent comment l'ignorance, la méconnaissance, le manque d'information et la perception des agents vaccinateurs par certaines mères concernant la maladie et de la vaccination contre la poliomyélite 
concourent en grande partie à leur hésitation ou leur réticence à la vaccination. Ainsi, il est aussi intéressant de noter la part des sources d'information et d'influence dans la réticence à la vaccination contre la poliomyélite.

\subsection{Les sources d'informations et d'influence}

Différentes sources d'informations ont été identifiées dans cette étude, à savoir: les sources formelles émanant des autorités de santé publique (télévision, radio, campagne de sensibilisation) et les sources populaires issues de la famille, de l'entourage, ou des amis (bouche à oreille). L'information obtenue par les différentes sources n'est pas sans influence directe sur la décision des mères. Pour certaines, c'est l'influence des proches, notamment des amies qui sont à l'origine de leur réticence à la vaccination.

"Une amie m'a dit qu'elle avait vu sur les réseaux sociaux que les gouttes qu'on donne à nos enfants étaient un moyen de contrôler nos enfants et de surtout de leur éviter de faire des enfants. Ils le font, parce que les noirs font beaucoup d'enfants, c'est pourquoi j'hésite à faire vacciner mon enfant. Vous savez, avec ce qu'on entend, vraiment, on ne sait jamais heiiin!!! »

D'autres affirmaient avoir été influencées par des membres de leur famille. Certaines étaient même en désaccord avec la décision de leur entourage, mais refusaient de faire vacciner leur enfant pour conserver les liens familiaux.

«Au début, c'est ma mère qui ne voulait pas que je fasse vacciner mon enfant. Selon elle, ce qu'on donne aux enfants n'est pas bon, donc c'est mieux de soigner l'enfant avec les plantes. Mais je n'étais pas d'accord avec elle, donc je faisais la vaccination de mon enfant sans qu'elle ne le sache. Après c'était ma belle-mère qui est rentrée dans la danse. Elle $m$ 'accusait de vouloir rendre son petit enfant stérile avec les vaccins bizarres des blancs. Alors, $j$ 'ai arrêté de faire vacciner l'enfant»

\section{Discussion}

Les facteurs associés à la réticence à la vaccination contre la poliomyélite mis en évidence dans notre étude sont relatifs à des aspects sociétaux et culturels. Ces facteurs combinés contribuent à expliquer les comportements de réticence exprimés par les mères à l'égard de la vaccination contre la poliomyélite dans le quartier Samaké de la commune d'Abobo. Les résultats dans leur ensemble montrent que les enquêtées ont une mauvaise compréhension et connaissance de la maladie et de la vaccination. L'ignorance et l'inquiétude concernant la sécurité du vaccin en lien avec les sources d'information contribuent aussi à cette réticence. 
Nos résultats sont superposables avec ceux retrouvés dans la littérature traitant des connaissances et croyance en lien avec la maladie et la vaccination et d'autres maladies comme la lèpre et le VIH.

En effet, selon Benin et al. (2006) et Bond et al. (1998), ces facteurs ont un rôle important dans la prise de décision car ils vont servir de base pour l'adoption de comportements. Ensuite, selon le Plan Stratégique National de Communication pour la vaccination de routine PNSC/ PEV 2014- 2016, très peu de mères et encore moins de pères connaissent le type des maladies évitables par la vaccination, le nombre de vaccinations qu'il faut faire, les rappels et le calendrier vaccinal. Aussi certains parents ne vont pas à la vaccination de routine et préfèrent les campagnes à la vaccination de routine parce qu'ils n'ont pas à se déplacer et peuvent se consacrer à leurs activités. Ceci montre que les contraintes liées aux services (temps d'attente, distances, horaires etc.) sont réelles et méritent d'être adressées.

Spoden (2016) a montré dans son étude que l'un des facteurs liés à la réticence contre la vaccination infantile chez les hésitants est leur mauvaise compréhension de la transmissibilité des maladies évitables par la vaccination. Ensuite selon Balinska \& Léon (2007) les maladies contre lesquelles les vaccins protègent ne sont plus connus du public et des soignants, il y'a une perte de la conscience collective par rapport à l'environnement infectieux dans lequel nous vivons et donc une relative dévalorisation de la vaccination.

Certains auteurs ont également documenté le fait que, malgré des connaissances nécessaires, certaines personnes ne mettent pas en pratique ce qu'il serait logique de faire (Cardia-Voneche \& Bastard, 1995). La décision de vacciner sa progéniture est influencée de différentes manières par ces facteurs et chaque mère réagit et interprète différemment les informations. Il convient de noter que ces facteurs ne sont pas indépendants les uns des autres, mais au contraire s'influencent mutuellement. Parfois, les mères peuvent être amenées à évaluer deux informations contradictoires, par exemple, des expériences graves suite à la vaccination rapportées par un tiers de personnes et contredites par les sources officielles ou par les professionnels de la santé. Ces déterminants ont déjà été documentés dans d'autres études, et l'importance du rôle du professionnel de la santé a été mise en avant (Bond et al., 1998 ; Freed et al., 2011 ; Leask, 2011 ; Poltorak et al., 2005). Certains auteurs comme Koffi et al. (2018) qui ont travaillé sur d'autres pathologies trouvaient dans leur étude que le diagnostic tardif et la réticence au dépistage de la lèpre étaient en lien avec les imaginaires sociaux négatifs associés à la lèpre, la durée d'hospitalisation et du traitement, la politique de dépistage non adaptée au contexte socioculturel et la méconnaissance des symptômes de la maladie par certains patients. Pour ce faire, Ils ont proposé des stratégies axées sur une sensibilisation accrue de la population ainsi que sur une politique de dépistage adéquate de cette maladie afin d'éradiquer la lèpre en Côte d'Ivoire. 
Plusieurs maladies comme le sida ont été, en effet, le lit de plusieurs écrits scientifiques. Par exemple, Gremy et al. (2002) ont mis en évidence dans leur étude l'évolution des connaissances, attitudes, croyances et comportements de la population générale face au VIH, une connaissance plus floue des modes de transmission de la maladie par la population. Cependant, les modes de protection contre le VIH-Sida étaient largement connus par la population depuis 1994, mais les mécanismes de la transmission ne semblent pas être complètement assimilés en 2001. À cette époque, plus de personnes interrogées pensaient que la contamination par le VIH était possible en utilisant une seringue neuve et lors des rapports sexuels avec préservatif. Les répondants ont été plus nombreux en 2001 qu'en 1998 à croire possible la contamination par « piqûre de moustique».

C'est dans ce même registre que Tia et al. (2016) ont montré dans leur étude que les représentations sociales du VIH chez les usagers de drogues (UD) en Côte-d'Ivoire étaient nourries par le sentiment de peur, d'amaigrissement puis de mort prochain et certain. Dans cette étude, les auteurs ont montré que les UD infectés du VIH-sida étaient perçus comme : «des balli », «des malchanceux», " des victimes de la maladie ». Ils étaient stigmatisés et rejetés dans la population générale comme dans la communauté des UD. Les auteurs ont ainsi conclu que la réticence au dépistage VIH chez les UD était due à la vision mortifère de cette maladie, à la crainte d'être stigmatisé, rejeté d'abord par la famille puis par la communauté UD et par ricochet, les ruptures socio-affectives.

\section{Conclusion}

$\mathrm{Au}$ terme de cette étude, nous retenons que les comportements de réticence à la vaccination contre la poliomyélite observés chez les mères dans le quartier Samaké de la commune d'Abobo (Abidjan) sont associés à un ensemble de facteurs sociétaux. Il ressort après l'analyse que la maladie de la poliomyélite et son vaccin sont perçus par les mères de diverses manières. La mauvaise compréhension et connaissance de la maladie et de la vaccination, l'ignorance, l'inquiétude concernant la sécurité du vaccin, le manque d'information concourent en grande partie à leur hésitation ou leur réticence à la vaccination contre la poliomyélite. D'autres facteurs comme l'influence des proches, ou des amies sont aussi à l'origine de leur réticence. Ce travail est alors une contribution dans la connaissance des principaux obstacles participant à la réticence à la vaccination contre la poliomyélite chez les mères. Ces résultats témoignent de l'intérêt de promouvoir des campagnes de sensibilisation sur la poliomyélite et aussi la mise en place de programmes de formations spécialisées pour tous les agents vaccinateurs afin de parvenir à une meilleure couverture vaccinale au cours des campagnes de vaccination de routine. 


\section{References:}

1. Antona, D. \& Guerin, N. (2005). L'éradication de la poliomyélite: où en est-on en 2010 ? Bulletin Épidémiologique Hebdomadaire, 48,489 -493.

2. Baliska, M-A. \& Léon, C. (2007). Opinions et réticences face à la vaccination. La Revue médecine interne 28, 28-32.

3. Benin, A., Wisler-Scher, D., Colson, E., Shapiro, E., \& Holmboe, S. (2006). Qualitative Analysis of Mothers'Decision-Making About Vaccines for Infants: The importance of Trust . Pediatrics, 117 (5), 1532-1541.

4. Blondel, B., Colbère - Garapin, F., Couderc, T., Wirotins, A., \& Guivel - Benhassine, F. (2000). Aspect moléculaire de la pathogenèse de la poliomyélite: les interactions poliovirus-cellules nerveuses. Virologie. 4(4): 30517.

5. Bond, L.T., Nolan, P., Pattison, \& Carlin, J. (1998). Vaccine diseases and immunisations: a qualitative study of mothers' perceptions of severity, susceptibility, benefits and barriers. Australian and New Zealand Journal of Public Health, 22 (4), 441 446.

6. Cardia-Voneche, L. \& Bastard, B. (1995). Préoccupations de santé et fonctionnement familial. Sciences sociales et santé, 13(1), $65-80$.

7. DCPEV (2017). Rapport de synthèse de la vaccination (Rapport de synthèse). $15 \mathrm{p}$

8. Freed, G., Clark, S., Butchart, A., Singer, D., \& Davis, M. (2011). Sources and Perceived Credibility of Vaccine-Safety Information for Parents . Pediatrics, 127, S107-S112.

9. Gremy, I., Chauveau, J., Vongmany, N., \& Beltzer, N. (2002). Les connaissances, attitudes, croyances et comportements face au VIH/ SIDA : évolution 1992-2001, adsp, 40, 25 -30.

10. Haut Conseil de la Santé Publique (2014). Avis relatif à la vaccination de rappel contre la poliomyélite pour certains voyageurs dans le contexte actuel d'urgence sanitaire décrétée par l'OMS. 11p.

11. Imbert, G. (2010). L'entretien semi - directif : à la frontière de la santé publique et de l'anthropologie, recherche en soins infirmiers, association de recherche en soins infirmiers (ARSI), 103/3, 23 -34.

12. Koffi, Y. O., Tia, F.Y., Kouamé, A., Yoro, B.M., \& Koné, D. (2018). Facteurs associés au retard du diagnostic et du Traitement de la lèpre en Côte d'Ivoire: cas des Patients sous traitement à l'institut Raoul Follereau D’Adzopé. Revue Espace Territoires Sociétés et Santé, Vol.1, 108 -119.

13. Leask, J. (2011). Target the fence-sitters . Nature, 473, $443-445$. 
14. Lefrançois, R. (1991). Dictionnaire de la recherche scientifique. (Les Éditions Némésis). Québec. 61p.

15. N'da, P. (2015). Recherche et méthodologie en sciences sociales et humaines: Réussir sa thèse, son mémoire de master ou professionnel, et son article (L'Harmattan). Paris. 107p.

16. Organisation Mondiale de la Santé (OMS) (2014). Poliovirus circulants dérivés d'une souche vaccinale confirmés et distincts au Soudan du Sud et à Madagascar - 2014. OMS. Consulté à l'adresse http://www.who.int/csr/don/14-november-2014-polio/fr/.

17. Organisation Mondiale de la Santé (OMS) (2015). Progrès accomplis en vue de l'éradication de la poliomyélite à l'échelle mondiale, 2014 2015. Relevé hebdomadaire, 90(21), 253 -259.

18. Organisation mondiale de la Santé (OMS) (2017). Couverture vaccinale. Consulté à l'adresse http://www.who.int/crs/don/16- juillet2017-polio/fr/

19. Poltorak, M., Leach, M., Fairhed, J., \& Cassell, J. (2005). MMR talk' and vaccination choice: An ethnographic study in Brighton. Social Science \& Medicine, (61), 709 -719.

20. Saraiva, A. (2014). La recherche qualitative dans le contexte carcéral. Stratégies, défis et pistes d'orientation. In Méthodes Qualitatives en Sciences Sociales et Humaines : perspectives et expériences. ( HorsSérie, 125 -130). Consulté à l'adresse http://www.recherchequalitative.qc.ca/revue/

21. Spoden, J. (2016). La vaccination infantile remise en questions, dans une pratique de médecine générale en fédération Wallonie-Bruxelles, Master complémentaire en médecine Université catholique de Louvain, Belgique. 90p

22. Tan Sien, H. L. (2016). La poliomyélite : une maladie ré-émergente ou en voie d'éradication? Université Angers, thèse de doctorat en pharmacie, 126p.

23. Tia, F.Y., Houndji, S.S.A, Dézé, C., \& Evanno, J. (2016). Analyse des représentations sociales du VIH-Sida chez les usagers de drogues à Abidjan (Côte-d'Ivoire), Présenté à Sciences Sociales et VIH-sida en Afrique Subsaharienne, Abidjan (Côte d'Ivoire). 159p

24. Tia, F.Y., Kouamé, A., Dézé, C., Evanno, J., \& Koné, D. (2017). Etude de l'inobservance à la thérapie antirétrovirale chez les usagers de drogues vivant avec le VIH (UDVVIH) à Abidjan. Revue Africaine (Nyansa-Pô), (N²3), 7 -28.

25. Yoro, B. M. (2012). Pluralisme thérapeutique et recours aux soins en milieu rural ivoirien: approche méthodologique. Recherches qualitatives, 31(1), 47 -61. 